\title{
Biocomposites Based on Biogenous Mineral for Inducing Biomimetic Mineralization
}

\author{
DIANA MARIA DRAGUSIN ${ }^{1,2}$, FILIS CURTI ${ }^{2}$, SERGIU CECOLTAN ${ }^{1}$, DANIELA SARGHIUTA ${ }^{2}$, LIVIA MARIA BUTAC ${ }^{1}$, \\ EUGENIU VASILE ${ }^{3}$, RODICA MARINESCU4*, IZABELA CRISTINA STANCU1,2,* \\ ${ }^{1}$ University Politehnica of Bucharest, Advanced Polymer Materials Group, 1-7 Ghe. Polizu Str., 011061, Bucharest, Romania \\ 2 University Politehnica of Bucharest, Faculty of Medical Engineering,1-7 Ghe. Polizu Str., 011061, Bucharest, Romania \\ ${ }^{3}$ University Politehnica of Bucharest, Department of Science and Engineering of Oxide Materials and Nanomaterials, 1-7 Ghe. \\ Polizu Str., 011061, Bucharest, Romania \\ ${ }^{4}$ Colentina Clinical Hospital Bucharest, 19-21 Stefan cel Mare Blvd., 020125, Bucharest, Romania
}

\begin{abstract}
In this work, we focused on the potential of biogenous mineral from Cuttlefish bone for inducing biomimetic mineralization with further biomedical applications. We demonstrate the formation of strong and flexible hydrogels via physical immobilization of cuttlefish bone powder in the presence of bovine gelatin and alginate. The properties of hydrogels loaded with biogenous mineral, with a commercial bone substitute, with commercial nano-hydroxyapatite and in situ generated hydroxyapatite. In vitro cell adhesion tests demonstrated that the new developed hydrogels showed a promising cell adhesion.
\end{abstract}

Keywords: cuttlefish bone, mineral phase, gelatin-alginate hydrogels, biocompatibility

Healing of fractures with loss of tissue, various types of bone defects, osteomyelitis, non-union, and arthrodesis continue to be major challenges in orthopedic surgery [1]. Autologous bone allografts have several excellent organizational characteristics, but the presence of immune rejection, diseases, and high costs for sample preparation, handling, and storage, make their usage a very restricted one [2].

Thus, scientists are constantly searching for alternative bone fillers [3]. Natural bio-products are becoming increasingly important for the preparation of new materials [4]. In the last years, cuttlefish bone has drowned an increased interest [5-7]. Due to its aragonite pillar-like structure, its feasibility as bone graft has been investigated in vitro [7]. It can be obtained easily and inexpensively from all the seas of the world, it is cheap [6], it can be shaped easily due to its morphology and mineral composition, it is compatible with other types of bone structure and it has high osteoinductive capacity [8] .

The mechanism governing the superior performances of hard tissue in contact with calcium based minerals ensue from the fact that bone cells are naturally accustomed with hydroxyapatite physiological environment, represented by the natural constituents of healthy bone and teeth [9-12]. Pursuant to the requirement of low cost and easy preparation with good performances (as mechanical and biological ones), cuttlefish bone represent a great alternative as mineral phase filler for polymeric materials [13]. One type of polymers that has been extensively applied for bone tissue regeneration includes hydrogels such as collagen, gelatin, alginates, chitosan and glycosaminoglycans [14-16]. Most hydrogels lack mechanical and/or thermal stability at physiological conditions, therefore cross-linking procedures and reinforcement with mineral phase have become practically inevitable due to their capacity to combine the tailorable properties of the bioactivity of the biopolymers with the properties of a calcium based mineral [16 - 18].

In this study, hydrogels based on gelatin and alginate, loaded with cuttlefish bone pow der have been synthesized and characterized. To prove the great potential of this biogenous mineral for bone applications, another series of hydrogels was prepared. In this case well known mineral phase was loaded into the hydrogels such as: commercially available bone substitute, nanohydroxyapatite and in situ generated nano-hydroxyapatite. The properties of all the materials were compared. The results lead to the conclusion that cuttlefish bone is indeed a promising bone filler due to the increased mechanical properties of the hybrid hydrogel, good biocompatibility, and tunable water affinity.

\section{Experimental part}

Materials and methods

Sodium alginate (alginic acid sodium salt from marine brown algae) with medium viscosity, gelatin from bovine skin (Type B, suitable for cell culture), glutaraldehyde (GA), calcium chloride $\left(\mathrm{CaCl}_{2}\right)$, sodium phosphate $\left(\mathrm{Na}_{2} \mathrm{HPO}_{4}\right)$ and nano-hydroxyapatite ( $\mathrm{n}$-Hap) were purchased from Sigma Aldrich. Glutaraldehyde and $\mathrm{CaCl}_{2}$ were used as cross-linkers. Cuttlefish bone (CB) was purchased from pet shops in Romania where it is sold as calcium supplement for birds. Eurocer 400 is a biphasic (hydroxyapatite $55 \%$ and tricalcium phosphate $45 \%$ ) commercial synthetic bone substitute (BS) developed by FH Orthopedics. $\mathrm{CaCl}_{2}$ and $\mathrm{Na}_{2} \mathrm{HPO}_{4}$ were used as mineralization precursors.

\section{Powder preparation}

The CB was cut in small cubes and extensively washed with double distilled water for 3 days at room temperature (RT). In a further step the CB cubes were dried and subsequently finely milled to a powder. The separation of the fine powder was performed through several decanting steps from water.

The same procedure of obtaining fine powder was applied for the commercial BS.

\section{Synthesis of the hybrid hydrogels}

The hybrid hydrogels were obtained through a threestep procedure: (1) dispersion of the mineral phase into the aqueous polymer solution, (2) physical gelation at $4^{\circ} \mathrm{C}$ and (3) cross-linking of the final compositions

\footnotetext{
* rodicamarinescu@yahoo.com; izabela.stancu@upb.ro
} 


\begin{tabular}{|c|c|c|c|c|}
\hline Sample & $\begin{array}{l}\text { Gelatin } \\
(w t \%)\end{array}$ & $\begin{array}{l}\text { Sodium } \\
\text { alginate } \\
\text { (wt } \%)\end{array}$ & \multicolumn{2}{|c|}{$\begin{array}{c}\text { Mineral } \\
\text { loading (wt } \%)\end{array}$} \\
\hline $\mathrm{FCO}$ & 5 & 1.5 & 0 & \\
\hline $\mathrm{FC1}$ & 5 & 1.5 & 2 & $\mathrm{CB}$ \\
\hline $\mathrm{FC} 2$ & 5 & 1.5 & 2 & BS \\
\hline $\mathrm{FC} 3$ & 5 & 1.5 & 2 & $\mathrm{n}-\mathrm{Hap}$ \\
\hline $\mathrm{FC4}$ & 5 & 1.5 & 2 & $\mathrm{Na}_{2} \mathrm{HPO}_{4}$ \\
\hline
\end{tabular}

Table 1

THE COMPOSITIONS OF THE INITIAL MIXTURES
(1) Firstly, gelatin Type B (GelB) aqueous solution ( $10 \%$ $\mathrm{wt}$ ) was prepared by the graduate dissolution of the protein in double-distilled water (ddw) at $40^{\circ} \mathrm{C}$. Sodium alginate (Alg) aqueous solution ( $3 \% \mathrm{wt}$ ) was prepared by the dissolution of the polysaccharide in ddw at $60^{\circ} \mathrm{C}$. Than, the mineral phase was dispersed in the GelB solution at $40^{\circ} \mathrm{C}$. In a subsequent step, the concentrated Alg solution was added and the homogenization continued by intensive stirring at $40^{\circ} \mathrm{C}$ followed by an additional step of homogenization using an ultrasound bath (Elma S $30 \mathrm{H}$, Elmasonic), for $15 \mathrm{~min}$ at $40^{\circ} \mathrm{C}$. A series of five mixtures with different compositions were prepared, including a control sample of GelB-Alg. The final compositions of the hybrid mixtures are depicted in table 1.

(2) In this intermediate stage, the mixtures were cast in Petri dishes to obtain thick membranes for further crosslinking and testing. The Petri dishes were kept at $4^{\circ} \mathrm{C}$ until complete physical gelation of the mixtures. This step was necessary also to avoid the mineral settling at the bottom of the casting molds.

(3) After gelation, the mixtures have been removed from the Petri dishes and have been immersed in aqueous GA$\mathrm{CaCl}_{2}$ solution for the crosslinking of the both polymeric components, for $24 \mathrm{~h}$. The non-reacted products been removed by extensive $d d w$ extraction, at $40^{\circ} \mathrm{C}$. By dissolution of $\mathrm{GA}(1 \mathrm{wt} \%)$ and $\mathrm{CaCl}_{2}(5 \mathrm{wt} \%)$ in ddw the cross-linking bath was obtained ( $\mathrm{pH}^{2}$ 7.4).

\section{Gel fraction analysis}

The gel fraction (GF, \%) was determined gravimetrically using the following equation (1):

$$
G F(\%)=\left({ }^{w_{f}} / w_{i}\right) \times 100
$$

where $w_{\text {i }}$ is the mass of the dried samples $(0.5 \mathrm{~cm} \times 0.5$ $\mathrm{cm})$ as they result from the reaction without purification and $w_{f}$ is the mass of the dried samples after extraction in $\mathrm{ddw}$ at $40^{\circ} \mathrm{C}$ for $24 \mathrm{~h}$.

\section{FTIR analysis}

The hybrid hydrogels were characterized using a J ASCO 4200 spectrometer equipped with a Specac Golden Gate attenuated total reflectance (ATR) device in the 4000-600 $\mathrm{cm}^{-1}$ wavenumber region.

\section{Evaluation of the water uptake capacity}

To evaluate the water affinity of the synthesized materials, swelling tests were performed (in triplicate) for each composition. The swelling degree (SD, \%) was calculated using equation 2 :

$$
S D(\%)=\left(w_{t}-w_{0} / w_{0}\right) \times 100
$$

where $w_{0}$ is the mass of the dried samples $(0.5 \mathrm{~cm} \times 0.5$ $\mathrm{cm})$ and $w_{t}$ is the mass of the hydrated hydrogels. All samples were allowed to swell at constant temperature $\left(37^{\circ} \mathrm{C}\right)$ in $d d w$, being removed at pre-established time periods, blotted and weighted, until constant mass was achieved. The equilibrium value is considered the maximum swelling degree (MSD, \%).

\section{Morpho-structural characterization}

Morphological and microstructural characterization of the hydrogels was performed through scanning electron microscopy (SEM) using QUANTA INSPECT F SEM device equipped with a field emission gun with $1.2 \mathrm{~nm}$ resolution and $a$ and with an X-ray energy dispersive spectrometer (EDAX). The analyses were performed on gold-sputtered dried samples. The samples were subjected to a dehydration procedure using a gradient of alcohol (ethanol). Micrographs were registered both on the surface and in the cross-section of the hybrid hydrogels.

\section{Evaluation of the mechanical behaviour}

Evaluation of mechanical properties of final mineral loaded hydrogels was performed using a CT3 texture analyzer with a $4500 \mathrm{~g}$ cell load (Brookfield Engineering) in compression mode. Cylindrical samples with a diameter of $13 \mathrm{~mm}$ and a thickness of $6 \mathrm{~mm}$, were placed on a plate and uniaxial pressed by the mechanical cell. The compression speed was set at $5 \mathrm{~mm} / \mathrm{min}$. A stressdeformation curve was plotted and the compression modulus was computed for each composition. Prior testing, the samples were brought to maximum swelling degree in ddw. The compression modulus was calculated from the slope of the initial linear part of the compression curve, at $5 \%$ strain.

In vitro evaluation of the cell behaviour

To determine the biocompatibility of synthesized samples, preliminary in vitro tests were performed. In vitro study was performed to assess osteoblast cell adhesion on the synthesized substrates. For this purpose, MG63 human osteosarcoma cells were used.

\section{(1) Cell seeding on hydrogels}

MG-63 human osteosarcoma cell-line (ECACC) were cultured in Dulbecco's Modified Eagle Medium (DMEM) (Sigma-Aldrich) supplemented with $10 \mathrm{wt} \%$ fetal bovine serum (FBS) (GIBCO), 1 wt $\%$ penicillin-streptomycin (Lonza) and $2 \mathrm{mM}$ glutamine. The cells were detached by trypsin/ EDTA (Merck Millipore) at 80\% confluence in the view of further use and seeded at a density of $10^{4}$ cells/ well for $24 \mathrm{~h}$, and incubated at $37^{\circ} \mathrm{C}$ in a humidified atmosphere with $5 \% \mathrm{CO}_{2}$. Cylindrical membranes with a diameter of $6.2 \mathrm{~mm}$, appropriately to the internal diameter of the well, were cut under sterile conditions. Then, the samples were centrifuged to remove existing spaces. After 24 hours of cultivation, non-adherent cells were removed using the culture medium.

\section{(2) MTT assay for cell viability}

The colorimetric MTT assay was performed to investigate the influence of the mineral phase on the viability of osteoblast cells. Cell viability was measured using an MTT reduction assay (3 [4,5-dimethylthiazol-2yl]-2,5-diphenyl tetrazolium bromide - Sigma Aldrich). 
To assess cell viability, MTT was added to a final concentration of $5 \mathrm{mg} / \mathrm{mL}$ in DMEM and cells were incubated for $3 \mathrm{~h}$ at $37^{\circ} \mathrm{C}$. The cells were incubated until the complete dissolution of the crystals. The supernatant was transferred to a new plate and the absorbance at a wavelength of $570 \mathrm{~nm}$ was measured on a TECAN M200PRO NANOCOUNT multiparametric plate reader.

\section{(3) Evaluation of cell morphology through SEM} investigations

The influence of mineral phases on the morphology and adhesion of the MG-63 cells on the analyzed membranes was further assessed through SEM analysis.

The protocol for SEM investigations involved washing the samples with phosphate buffered saline (PBS) three times to remove non-adherent cells and the culture medium. Subsequently, $100 \mu \mathrm{L}$ of $4 \mathrm{wt} \%$ paraformaldehyde solution was added, and were incubated for 1 hour at $37^{\circ} \mathrm{C}$. Removal of paraformaldehyde was carried out by washing with PBS for three times ( $100 \mu \mathrm{L})$, followed by the procedure of dehydration using gradient of alcohol (ethanol).

\section{Results and discussions}

In the present study, we report on the synthesis and the physical characterization of crosslinked hybrid hydrogels based on natural originated polymers (GelB and Alg) and phase in the aqueous solutions of biopolymers, and then the cross-linking resulting in the hybrid hydrogels. Additionally, in case of the last composition (FC4) this synthesis method has a double contribution: on one side the cross-linking of the biopolymers, and on the other side the in situ generation of a mineral phase, namely $n$-Hap.

\section{Gel fraction analysis}

The investigation of the hybrid hydrogels developed in this study started with the estimation of GF. The parameter quantifies the success of the reaction while indicating the migration of the hydrosoluble species in water. Therefore, the materials were incubated at $37^{\circ} \mathrm{C}$ in ddw for $24 \mathrm{~h}$. The results were estimated using equation (1) and the obtained GF values are summarized in figure 1 .

All synthesized hydrogels presented high GF values (higher than 90\%), indicating: (i) mass conservation during the treatment, and (ii) water insolubility of the formed bicomponent systems. This applied also for the control sample, proving the efficiency of the applied cross-linking method with respect to the synthesis of the corresponding bicomponent system. The lowest value of the gel fraction was assessed for the material having the mineral generated in situ $(90.71 \% \pm 1.95)$ and the highest value was assessed for the hydrogel containing commercial nHap $(95.78 \% \pm 0.67)$. All the other composition presented values of the GF ranging between $91.32 \%$ and $94.97 \%$.

\section{Precursors}

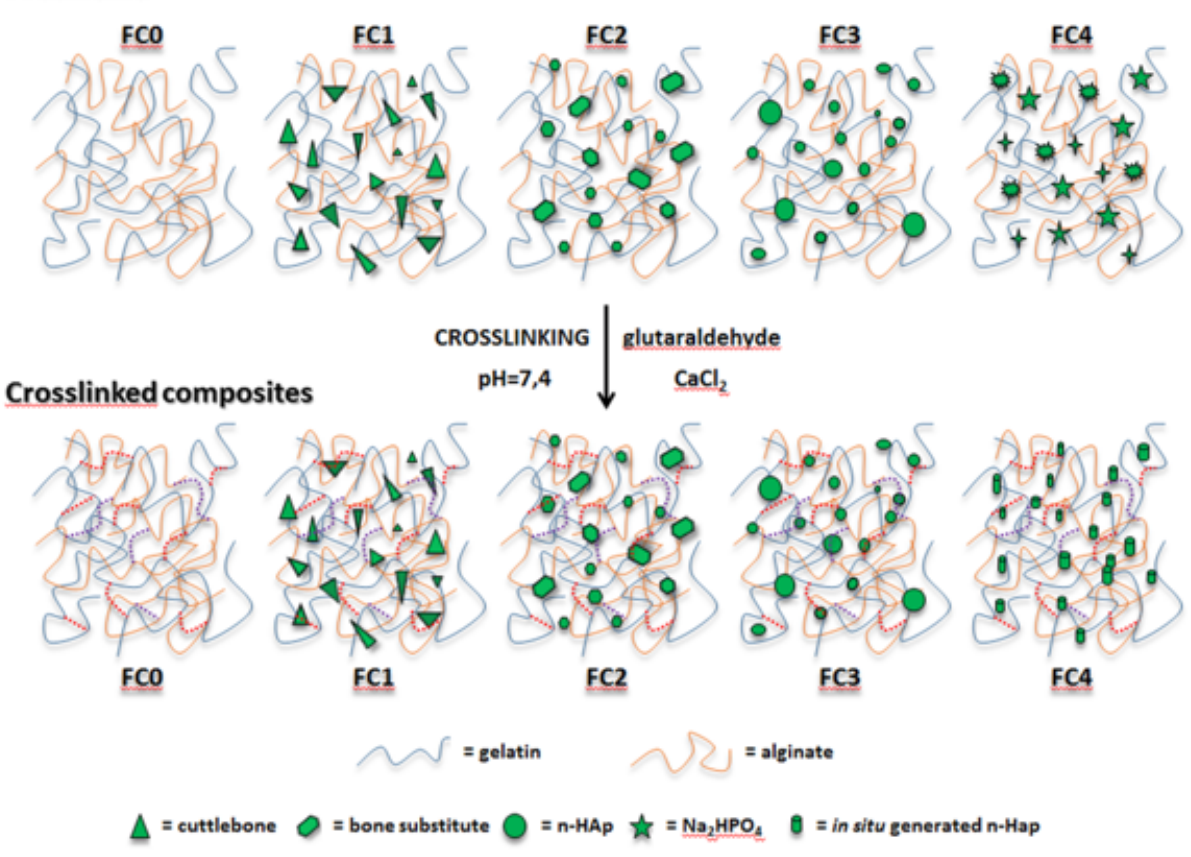

Scheme 1. Scheme representing the synthesis of FCO: control GelB/Alg hydrogel, $\mathrm{FCl}$ : CB-GelB/Alg hybrid hydrogel, FC2: BS-GelB/Alg hybrid hydrogel, FC3: n-Hap-GelB/Alg hybrid hydrogel, FC4: in situ generated n-Hap GelB/Alg hybrid hydrogel different types of mineral. The novelty of the study is the introduction of $C B$ which presents outstanding characteristics with potential in filling and repairing bone defects, that are currently under exploited. To support this affirmation, we compared the properties of hybrid hydrogels containing as reinforcement filling not only $C B$, but also commercially available BS, $\mathrm{n}$-Hap and in situ generated $\mathrm{n}$-Hap (having as precursors $\mathrm{Na}_{2} \mathrm{HPO}_{4}$ and $\mathrm{CaCl}_{2}$ ) and a control hydrogel GelB-Alg. The schematic synthesis of the materials is presented in scheme 1.

The polymeric system, representing the organic phase of the synthesized materials, is studied by our group for various applications from skin grafts to bone substitute [1618].

The synthesis of these biomaterials is a simple method which mainly involves a good dispersion of the mineral

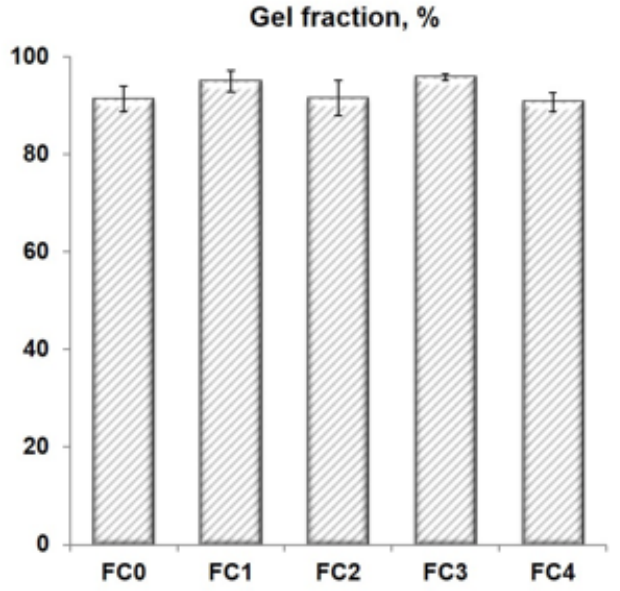

Fig. 1. GF average values for the hybrid hydrogels FCO - FC4 


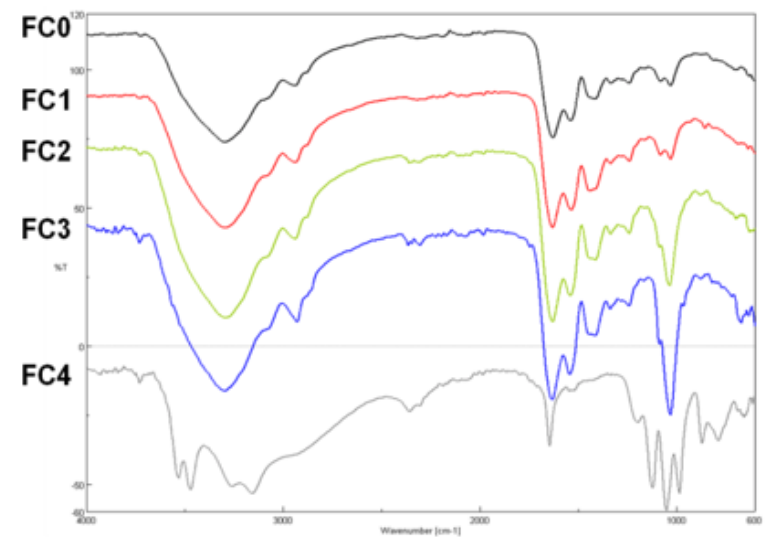

Fig. 2. FTIR spectra recorded on control sample FCO and on samples FC1-FC4

\section{FTIR analysis}

The study was further continued with FTIR analyses. To confirm the presence of all the components in the hydrogels, the samples FC1-FC4 were screened with respect to the functional group attributes for each component (fig. 2). The obtained results were compared to those of FCO.

All the hydrogels have presented typical $\mathrm{O}-\mathrm{H}$ and $\mathrm{N}-\mathrm{H}$ vibrations at about $3300-3400 \mathrm{~cm}^{-1}$, due to the abundance of such groups in the Gel and alginate components. Amide I and II (at $1630 \mathrm{~cm}^{-1}$ and $1545 \mathrm{~cm}^{-1}$, respectively) are also visible due to the peptide.

As it is well known, typical apatite presents $\mathrm{OH}$ stretching vibration at $3570 \mathrm{~cm}^{-1}$ and $\mathrm{PO}_{4}{ }^{3-}$ vibration modes at 1096, 1036 and at $960 \mathrm{~cm}^{-1}$, respectively. All these peaks are, as expected, visible for samples FC2, FC3 and FC4, where $n$-Hap is incorporated in the hydrogels (physically from the initial mixtures, or generated in situ as is the case of F(4).

The cuttlebone shows two peaks of the carbonate vibration at 700 and $713 \mathrm{~cm}^{-1}$ which are attributed to aragonite [19]. The peak at $854 \mathrm{~cm}^{-1}$ is also characteristic of aragonite. Preservation of aragonite, a meta-stable form of calcium carbonate, rather than the more stable calcite form, indicate the cuttlebones have undergone minimal diagenesis and thus had a greater potential to preserve original organics. Peaks at $1650 \mathrm{~cm}^{-1}$ (Amide I) and 1544 $\mathrm{cm}^{-1}$ (Amide II) in cuttlefish spectra indicate organics consistent with $\beta$-chitin (data not presented).

\section{Evaluation of the water uptake capacity}

For a scaffold intended to be used as potential implantable material, the capacity to absorb body fluids and to transfer nutrients represents a key element. Many factors can influence the water uptake capacity of a hydrogel, including the hydrophilicity of the constituents, the chemical composition and finally, by the cross-linking degree. Any variation of the composition of the polymeric network can control the swelling behavior. The water affinity of all types of developed hydrogels was studied through incubation in $d d w$, at $37^{\circ} \mathrm{C}$, while monitoring the water uptake at regular time points. SD was calculated using equation (2) and the results are summarized in Figure 3. The results obtained for MSD show high values in the interval 311 - 509\%. The values of the MSD for the control sample, $F C 0$, and the hydrogel containing CB, $F C 1$, are the highest ones ( $443 \%$, and $509 \%$ respectively). These results conclude that the water affinity degree is highly dependent on the mineral type. The high-water uptake for FCO can be correlated with the initial hollow structure of the $C B$,

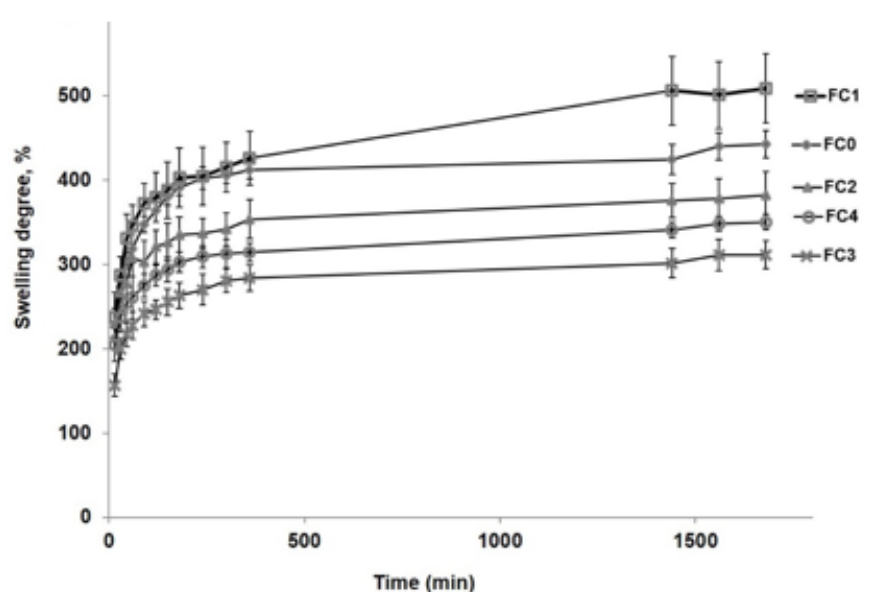

Fig. 3.The influence of the composition and mineral type on the water affinity of the hydrogels

therefore allowing the uptake of a larger amount of aqueous solution. All the other materials presentMSD lower than $400 \%$. The composition with the best dimensional stability was the composition that incorporate commercial n-Hap (311\%).

This elicits important variations of the swelling behavior of the bicomponent materials upon modification of the mineral type. The results obtained in the swelling study indicate variations of elasticity for the synthesized hybrids that will be further investigated through mechanical studies.

\section{Morpho-structural characterization}

SEM was used to assess the morphology of the materials after the cross-linking. The most important feature was the homogenous dispersion of the mineral into the entire block of the hydrogels. Representative images with respect to these parameters are shown in figure 4.

SEM characterization reveals a good dispersion of the mineral phase into the polymer matrix (fig. 4, panel (1)). The control sample FCO presents a compact structure (fig. 4 (A1)) but an interesting internal, fine, porosity (fig. 4(A2)) most likely due to the dehydration phenomenon with gradient of ethanol. In figure 4 (B1 and B2) it can be observed the dispersion of the $C B$ into the polymeric structure. The shape and the dimensions of the $C B$ particles are irregular and, when compared with the other minerals used the particles have important higher dimensions of even $10^{3}$ times higher. The internal structure of the CB is a very complex one as shown in figure 5 .

SEM shows the tunnel-like microstructures of cuttlebones with the trabeculae in the holes the; trabeculae consist of the thin layer cuttings. It is expected that even after the grinding of the mineral some internal porosity will be maintained (aspect which was pursued). This affirmation also correlates with the water affinity of the hydrogel loaded with CB. Due to the internal porous structure of the mineral, the water uptake is higher than of the rest of synthesized structures.

With concern to the rest of the mineral types, the morphology of the corresponding composites present a more uniform shape and dimensions of the inorganic phase. Thus, for sample FC2, where BS was used, the granular aspect of the mineral is present into the entire polymeric matrix. For sample FC3, loaded with n-Hap, the dimensions of the particle are ranging between 40 and $100 \mathrm{~nm}$. In case of sample FC4, with generated n-Hap during the crosslinking step, the structure of the mineral is typical with needle like aspect. This mineral phase has a very intimate 

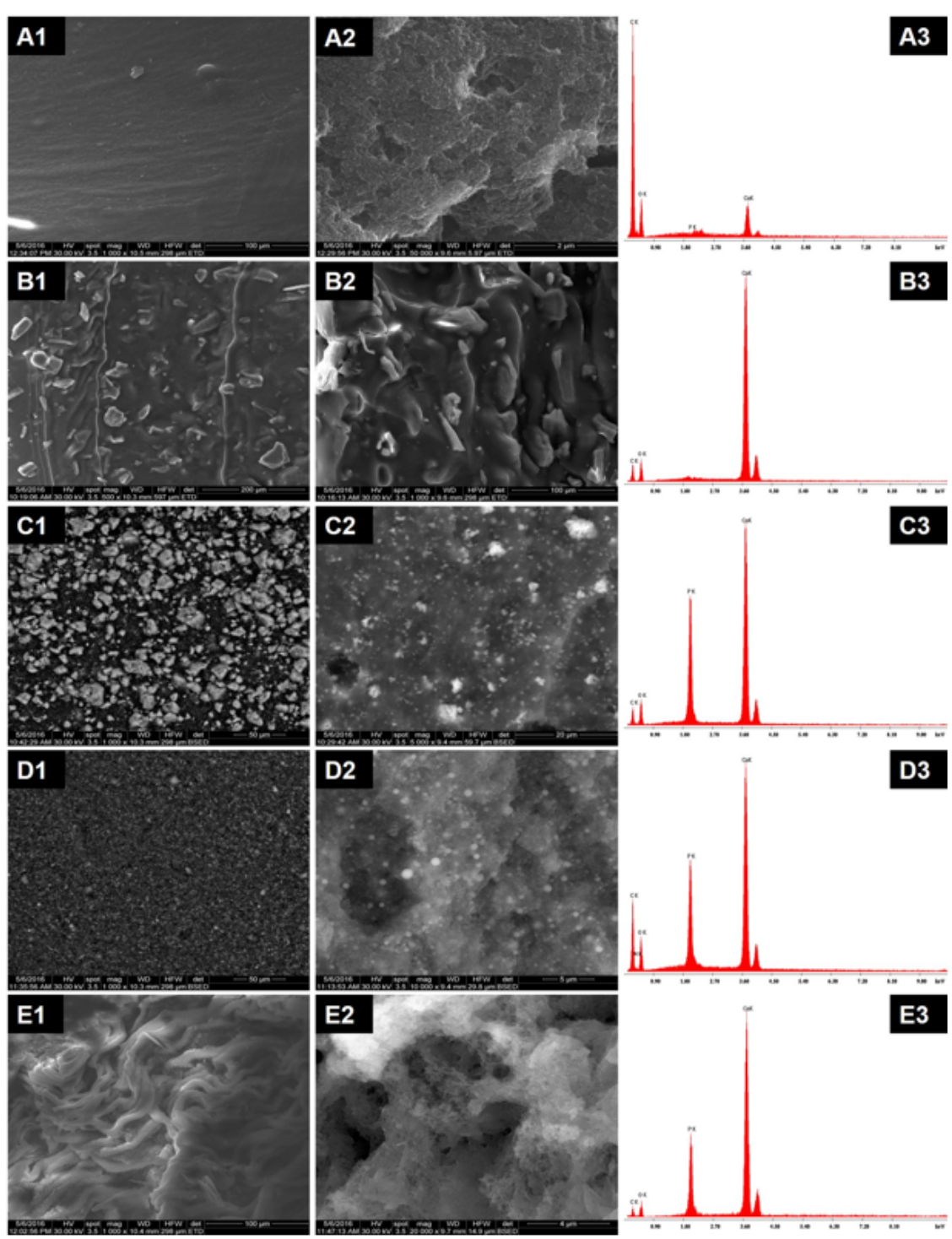

A3

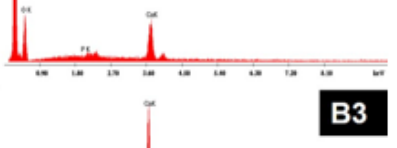

C3
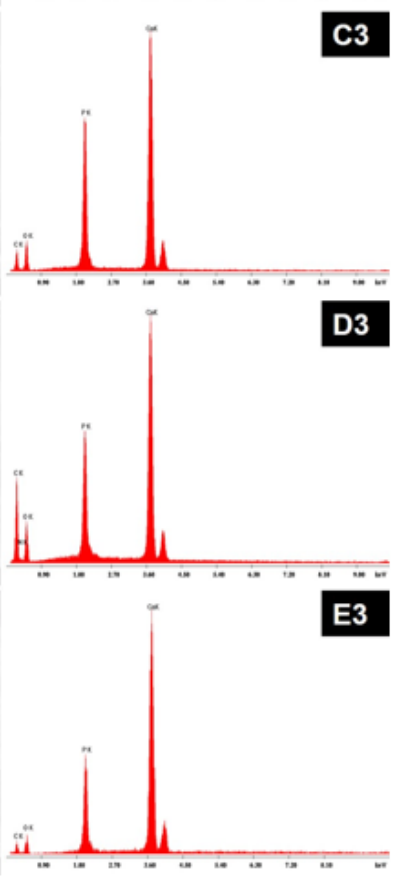

Fig. 4. Morphology of the of the control sample FCO and hybrid hydrogels as obtained through SEM (panel (1) - top view, panel (2) - side view) and EDAX spectra

(panel (3))

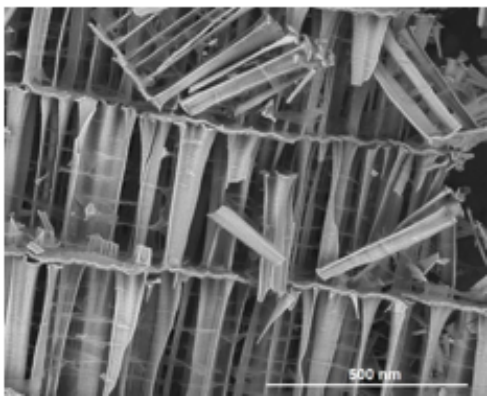

Fig. 5. SEM morphology of the CB nanosized grains organized in coral-like clusters.Nanometric pores are slso visible contact with the polymer network, which presents itself as filaments covered with n-Hap.

Further, EDAX detected the presence of calcium in all the materials. FCO presents a small amount of calcium due to the cross-linking method which uses $\mathrm{CaCl}_{2}$ as crosslinking agent for Alg. Regarding all the other materials FC1$\mathrm{FC4}$, Ca and P with different ratios, are detected all over the materials. The only sample that has irrelevant traces of $P$ is $F C 1$. This ensue since $C B$ is mainly carbonate in its aragonite form.

For samples FC3 and FC4 the EDAX spectra are very similar, proving the fact that even the mineral that was in situ generated during the cross-linking is n-Hap.

\section{Mechanical properties}

Regarding the mechanical properties of the hybrid hydrogels, compression tests were performed on the fully hydrated samples, showing that the material with $C B$ content has the highest compression modulus, which equals a higher stiffness. In case of the hydrogels loaded with $\mathrm{BS}$ and $\mathrm{n}$-Hap the mechanical properties are similar with the ones of the control samples. This may conclude that the only type of mineral that brings an important improvement of the stiffness of the GelB-Alg system is the $\mathrm{CB}$ as depicted in figure 6 .

The results obtained on the compression tests for samples FCO, FC2, FC3 and FC4 are in agreement with the data registered in the water affinity tests, stating for the reinforcing effect of mineral phase as proven by the increase of the compression modulus from $71 \pm 13 \mathrm{kPa}$ for FCO to $82 \pm 12 \mathrm{kPa}$ for FC3. The only lack of correlation between the swelling behaviour and the compression modulus was found for $\mathrm{FCl}$, which was the highest (119 $\pm 13 \mathrm{kPa}$ ). Even so, the explanation converges from the internal structure of the $\mathrm{CB}$, able to fill up with aqueous medium the internal pores, without having a plasticizing effect on the polymeric matrix.

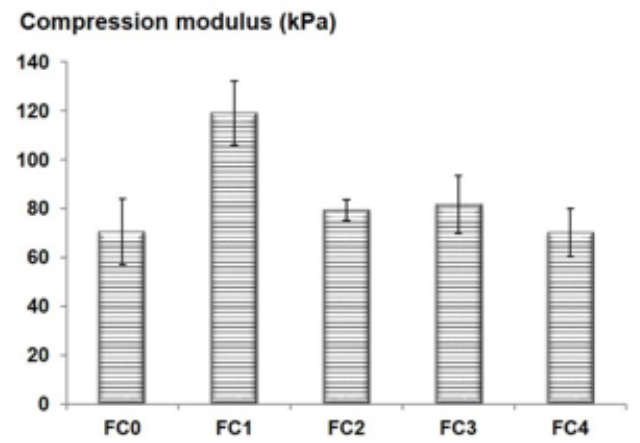

Fig. 6. The compression modulus of the hybrid materials and control sample

In vitro evaluation of the cell behaviour

Preliminary biological study pursued the effects of mineral phases loaded into hydrogels on osteoblast adhesion on the surface of synthesized hydrogels membranes. Figure 7 illustrates the number of viable cells adherent on the hydrogel samples as determined by MTT 
assay after one day of incubation, relative to the tissue culture plate control.

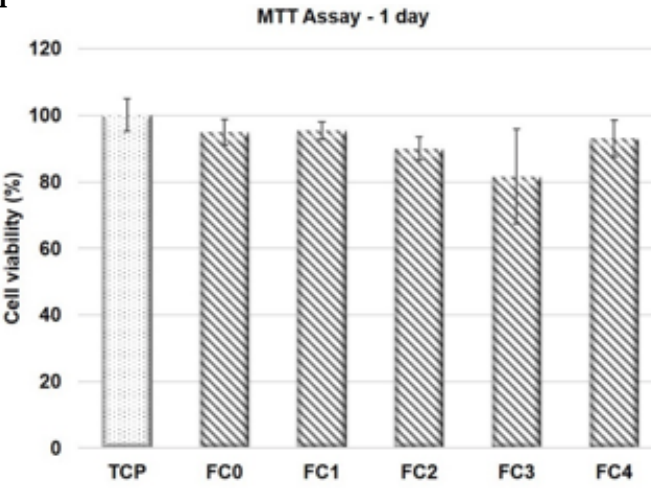

Fig. 7. Cell viability tests performed by MTT assay on FCO - FC4, $n=3$. Percentage of viable cells relative to the tissue culture plate control after 1 day
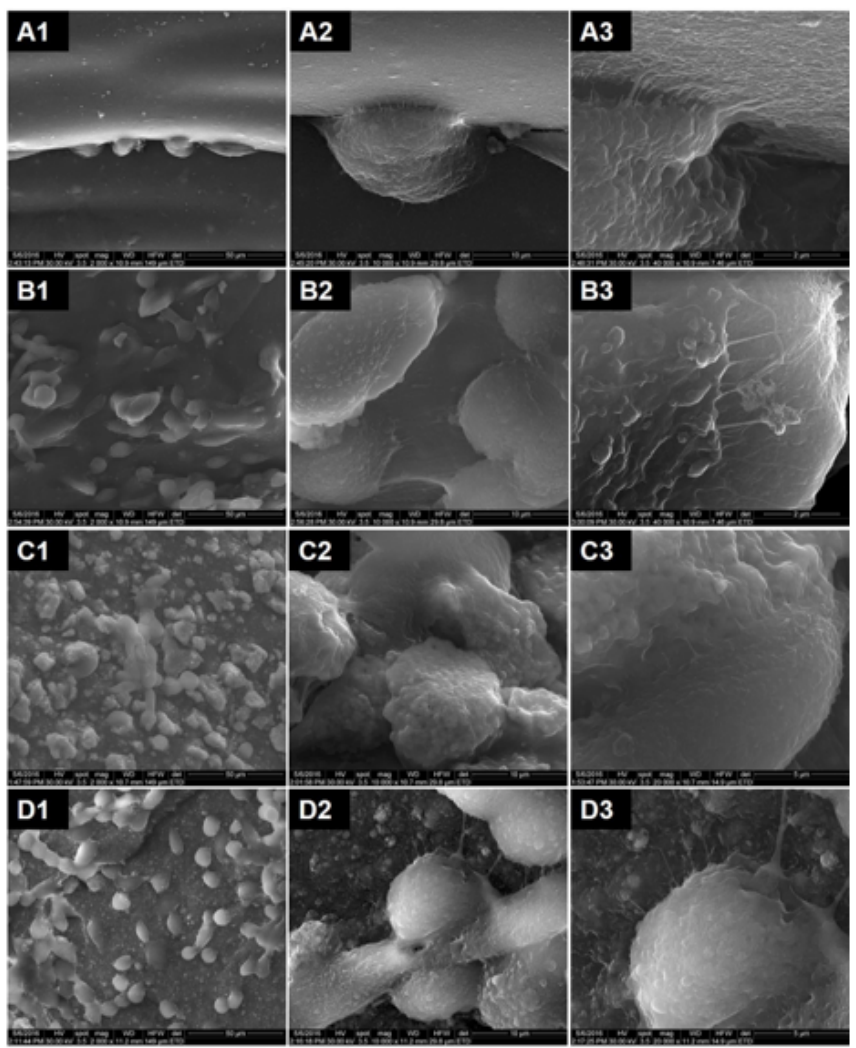

E1
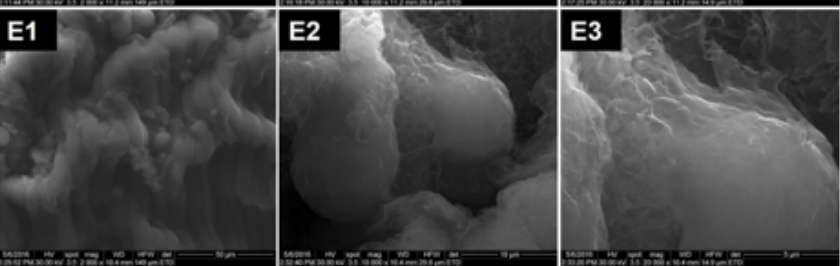

Fig. 8. SEM micrographs of MG63 cells at $24 \mathrm{~h}$ post seeding on FCO - FC4 hydrogels: series (1): $2000 \mathrm{x}$ magnification presenting the good spreading of the cells on the surface of the hydrogels; series

(2): 10,000 x magnification showing the preferential adhesion on the mineral particles; series (3): images showing cells' membrane protrusions

After one day, the number of cells on the FCO hydrogel was $94.9 \pm 3.8 \%$, on $\mathrm{FCl}$ was $95.4 \pm 2.7 \%$, on FC2 was $89.9 \pm 3.4 \%$, on FC3 was $81.7 \pm 14.2 \%$ and on FC4 was 93 $\pm 5.6 \%$, in comparison with $100 \pm 4.9 \%$ of the control culture. The recorded values for $\mathrm{FCO}$ and $\mathrm{FCl}$ are quite similar, with a quite small increase of the cellular viability for the hydrogel embedding CB. This result is an important indication of low cytotoxicity of the biocomposites and a promising cell activity which suggests that cuttlefish bone has great potential for use as a biomaterial.
To investigate the effect of mineral loading on the adhesive properties of the hybrid hydrogels, the morphological features of MG-63 cells were evaluated using SEM at $24 \mathrm{~h}$ post-seeding (fig. 8).

One day after seeding, MG-63 cells with both flat spindle shaped morphology and round morphology, specific for attached and non-attached cells, were observed on all the scaffolds. In case of the attached cells the membranes presented an intimate contact with the surface of the hydrogels. Even more, it can be observed the preference of the cells for the areas where they could encounter mineral phase. Also, the cells generated filopodia which are thin, actin-rich plasma-membrane protrusions that function as antennae for cells to probe their environment, filaments that can be seen mostly in case of the hydrogels with mineral loadings. These findings are promising for the use of $\mathrm{CB}$ composite in bone regeneration.

\section{Conclusions}

In summary, this study presents the potential of cuttlefish bone to be used as biogenous mineral with applications for bone tissue regeneration. We compared the properties of a hybrid hydrogel based on gelatin and alginate loaded with CB and with other well-known and extensively studied types of minerals (commercially available bone substitute and nano-hydroxyapatite and in situ generated $n$-Hap). It could be observed a significant improvement of the mechanical properties in case of the hydrogel loaded with CB. Also in vitro cell adhesion tests demonstrated that this material showed a promising cell activity.

Therefore, we consider that the hybrid hydrogels GelBAlg-CB may be generated as bulk or porous constructs with interesting properties that are useful when aiming at bone repairing. The described synthesis method could enable us to obtain a wide range of mineral loadings. This system will be further developed.

Acknowledgement: The authors would like to acknowledge the financial support from the project 201/2014, Zettaskin.

\section{References}

1. OH T., RAHMAN M.M., LIM J.H., PARK M.S., KIM D.Y., YOON J.H., KIM W.H., KIKUCHI M., TANAKA J., KOYAMA Y., KWEON O.K., J. Vet. Sci, vol. 7, no.1, pp. 73-77, 2006.

2. GHODASRA J.H., DALEY E.L., HSU E.L., HSU W.K., Eur. Spine. J., vol. 23, no.2, pp. 426-434, 2014.

3.DOGAN E., OKUMUS Z., Vet. Med., vol. 59, no.5, pp. 254-260, 2014. 4. POMPE W.; RODEL G.; WEISS H.J ., MERTIG M., Bio-Nanomaterials: Designing Materials Inspired by Nature; Wiley-VCH: Weinheim, Germany, 2013.

5.ROCHA J.H.G., LEMOSA.F., AGATHOPOULOSS., KANNAN S., VALERIO P., FERREIRA J .M.F., J. Biomed. Mater. Res. A, vol. 77, no.1, pp. 160168, 2006.

6.YILDIRIM O.S., OKUMUS Z., KIZILKAYA M., OZDEMIR Y., DURAK R., OKUR A., CAN J. Anal. Sci. Spect., vol. 52, pp. 270-275, 2007.

7. IVANKOVIC H., GALLEGO FERRER G., TKALCEC E., ORLIC S., IVANKOVIC M., J. Mater. Sci.: Mater. Med. vol. 20, no.5, pp.1039-1046, 2009.

8. MURUGAN R., RAMAKRISHNA S., PANDURANGA RAO K., Mater. Lett., vol. 60, no.23, pp. 2844-2847, 2006.

9. MUNARINA F., PETRINI P., GENTILINI R., PILLAI R.S., DIRÈ S., TANZI M.C., SGLAVO V.M., Int. J. Biol. Macromolec., vol. 72, pp. 199-209, 2015.

10.WATSON B.M., VO T.N., ENGEL P.S., MIKOS A.G., Ind. Eng. Chem. Res., vol. 54, no.42, pp. 10206-10211, 2015.

11. BJ ORNOY S.H., BASSETT D.C, UCAR S., STRAND B.L., ANDREASSEN J.-P., SIKORSKI P., Acta Biomater., vol. 44, pp. 254-266, 2016. 
12. LUNGU, A., FLOREA N,.M., DAMIAN, C.M., IOVU, H., Mat. Plast., 48, no.3, 2011, p. 209

13. ROCHA, J.H.G., LEMOS, A.F., AGATHOPOULOS, S., VALERIO, P., KANNAN S., OKTAR F.N., FERREIRA J.M.F., Bone, vol. 37, pp. 850-857, 2005.

14. JIN, R., TEIXEIRA, L.S., DIJ KSTRA, P.J ., VAN BLITTERSWIJ K, C.A., KARPERIEN, M., FEIJEN J., J. Control. Release, vol. 152, no.1, pp. 186-195, 2011.

15. JIN R., TEIXEIRA L.S., DIJKSTRA P.J., VAN BLITTERSWIJK C.A., KARPERIEN M., FEIJEN J., Biomaterials, vol. 31, no.11, pp. 3103-3113, 2010.
16. PETRE, D., CECOLTAN, S., SERAFIM, A., LUNGU, A., DRAGUSIN, D. M., STAN, E. G., TUCUREANU, C., VASILE, E., SALAGEANU, A., ISTODORESCU, M., IOVU, H., STANCU, I. C., Mat. Plast., 53, no. 2, 2016, p. 269

17. STANCU I.C., DRAGUSIN D.M., VASILE E., TRUSCA R., ANTONIAC I., VASILESCU D.S., J. Mater. Sci.: Mater. Med., vol. 22, no. 3, pp. 451460, 2011.

18. STANCU I.C., LUNGU A., DRAGUSIN D.M., VASILE E., DAMIAN C., IOVU H., Soft Mater., vol. 11, no. 4, pp. 384-393, 2013.

19. FLOREK M., FORNAL E., GÓMEZ-ROMERO P., ZIEBA E., PASZKOWICZ W., LEKKI J., NOWAK J., KUCZUMOW A., Mater. Sci. Eng. C., vol. 29, no. 4, pp. 1220-1226, 2009

Manuscript received: 11.12 .2016 